\title{
SIMULATING THE MECHANICS OF MYOCARDIAL TISSUE USING STRONGLY SCALABLE PARALLEL ALGORITHMS
}

\author{
Christoph M. Augustin ${ }^{1}$ and Gernot Plank ${ }^{1,2}$ \\ ${ }^{1}$ Institute of Biophysics, Medical University of Graz, Harrachgasse 21, 8010 Graz, Austria \\ ${ }^{2}$ Oxford e-Research Centre, University of Oxford, Keble Road 7, OX1 3QG Oxford, UK \\ christoph.augustin@medunigraz.at
}

\begin{abstract}
Due to preferential orientations of fibers, such as collagen or myocytes, the modeling of the mechanics of myocardial tissue leads to anisotropic and highly nonlinear material models. For micro-anatomically realistic geometries the computational effort to handle these sophisticated models is very challenging and demands the usage of strongly scalable parallel algorithms. In this context we mention two possible approaches and show numerical examples.
\end{abstract}

Keywords: Mechanics, biological tissues, active stress, myocardium, high performance computing, FETI, multigrid

\section{Modeling}

For the numerical simulation of the elastic behavior of biological tissue we consider the stationary equilibrium equations

$$
-\operatorname{Div} \mathbf{F S}(\mathbf{u}, \mathbf{x})=\mathbf{0}, \quad \text { for } \mathbf{x} \in \Omega
$$

to find a deformation $\mathbf{u}$ in a domain $\Omega$, in our case the myocardium. In (1) we introduce the deformation gradient $\mathbf{F}=\mathbf{I}+\operatorname{Grad}(\mathbf{u})$ and the second Piola-Kirchhoff stress tensor S. In addition, we incorporate Dirichlet and Neumann boundary conditions to describe fixed displacements and surface tractions on $\Gamma=\partial \Omega$. The total stress tensor $\mathbf{S}$ is obtained by an addition of a passive and an active stress part

$$
\mathbf{S}=\mathbf{S}_{p}+\mathbf{S}_{a}
$$

which will be described in the following.

\section{Passive Stress}

For the derivation of a constitutive equation for the passive stress tensor $\mathbf{S}_{p}$, we introduce the strain-energy function $\Psi(\mathbf{C})$. Using this, we can state the constitutive equation

$$
\mathbf{S}_{p}=2 \frac{\partial \Psi(\mathbf{C})}{\partial \mathbf{C}}
$$

where $\mathbf{C}=\mathbf{F}^{\top} \mathbf{F}$ is the right Cauchy-Green tensor.

The specific form of the strain-energy function varies from material to material, but in the case of myocardial tissue we use the common structure

$$
\Psi=\Psi_{\text {vol }}+\Psi_{\text {iso }}+\Psi_{\text {aniso }} .
$$

Here, the volumetric contribution $\Psi_{\mathrm{vol}}$ is used to handle the nearly incompressibility condition which is typical for most biological tissues.

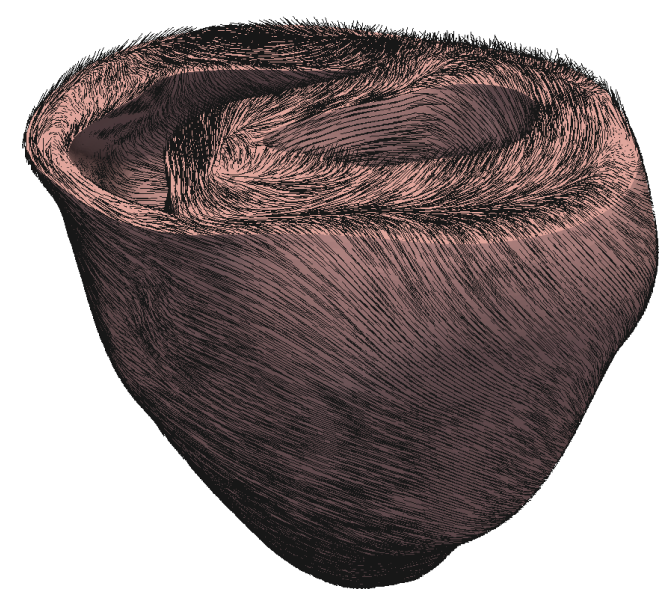

Figure 1: Right and left ventricle of a rabbit heart where the black lines indicate the myocyte network. The mesh has 547680 vertices and 3073529 tetrahedral elements.

The isotropic component of the material, i.e. the underlying non-collagenous and non-muscular matrix, is modeled using the well-known Demiray model ( $\left.\Psi_{\text {iso }}\right)$.

Due to preferential orientations of fibers (Fig. 1) the modeling of myocardial tissue leads to an anisotropic material behavior, which is incorporated in $\Psi_{\text {aniso. }}$. From the histology of the myocardium we observe two main fiber networks, myocytes $(f)$ and collagen fibers $(s)$. Following Holzapfel and Ogden [1] the anisotropic contribution is then modeled using an exponential structure of the type

$$
\Psi_{\text {aniso }}=\frac{a_{i}}{2 b_{i}}\left\{\exp \left[a_{i}\left(I_{i}\left(\mathbf{C}, \mathbf{f}_{i}\right)-1\right)^{2}\right]-1\right\},
$$

for each fiber group $i=f, s$. The invariants $I_{i}\left(\mathbf{C}, \mathbf{f}_{i}\right)$ represent the stretch in a fiber direction $\mathbf{f}_{i}$ and $a_{i}, b_{i}$ are positive parameters. An additional term, structurally comparable to (5), models the interaction between the two fiber networks.

\section{Active Stress}

The active stress component is generated by the electrical activation in the myocardial tissue. In this context we make use of the bidomain equations which describe the spread of electrical activation in the heart, e.g., see [2], to compute the transmembrane voltage $V_{m}$. A scalar-valued active stress term $S_{a}$ is then retrieved by

$$
S_{a}=h\left(V_{m}, \eta, \mathbf{u}\right),
$$

where the function $h$ and the state variables $\eta$ depend on the respective cell model, see CellML (www.cellml.org). 
Hence, the active contribution to the second Piola-Kirchhoff stress tensor is obtained by

$$
\mathbf{S}_{a}=S_{a} I_{f}^{-1}\left(\mathbf{f}_{f} \otimes \mathbf{f}_{f}\right)
$$

where $\mathbf{f}_{f}$ is the main orientation of the myocyte network and $I_{f}$ was already introduced in (5). For more details concerning the modeling of cardiac materials see $[1,3]$.

\section{Numerical Methods and Results}

In order to obtain a numerical solution of Eqs. 1-7 we use variational and finite element techniques. To solve the nonlinear system we perform a linearization to apply Newton's method:

$$
\mathbf{K}^{\prime}\left(\underline{u}^{k}\right) \Delta \underline{u}=\underline{\mathcal{F}}\left(\underline{u}^{k}\right)-\underline{K}\left(\underline{u}^{k}\right), \quad \underline{u}^{k+1}=\underline{u}^{k}+\Delta \underline{u} .
$$

In this equation $\mathbf{K}^{\prime}\left(\underline{u}^{k}\right)$ is the tangent stiffness matrix generated by using finite element techniques, $\underline{u}^{k}$ is the displacement vector at Newton step $k$ and $\underline{\mathcal{F}}\left(\underline{u}^{k}\right)$ captures the loads acting on the boundary of $\Omega . \Delta \underline{u}$ is the update to be calculated from the linearized system $(8)_{1}$. For a fine grid, resulting from detailed geometries, this linearized system of equations involves a very large number of degrees of freedom, which can not be handled by direct solving methods or single core algorithms in a reasonable time.

Hence, we have to apply parallel solving methods such as the finite element tearing and interconnecting (FETI) method, see [4], or algebraic multigrid (AMG) methods, see [5], which both share the same principle: a decomposition of the domain $\Omega$ into subdomains (Fig. 3).

The strong scalability of the implementation up to 256 cores is illustrated by means of Fig. 2 on the example of the FETI method. We obtain similar results using the AMG approach.

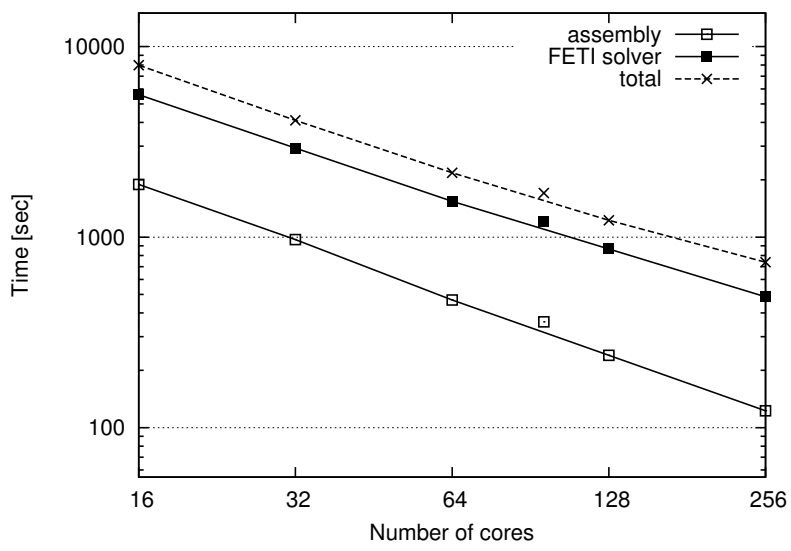

Figure 2: Computational times for a passive myocardium simulation using the FETI approach and the heart mesh shown as in Fig 1. The times are measured for 5 load steps with 6 Newton steps each.

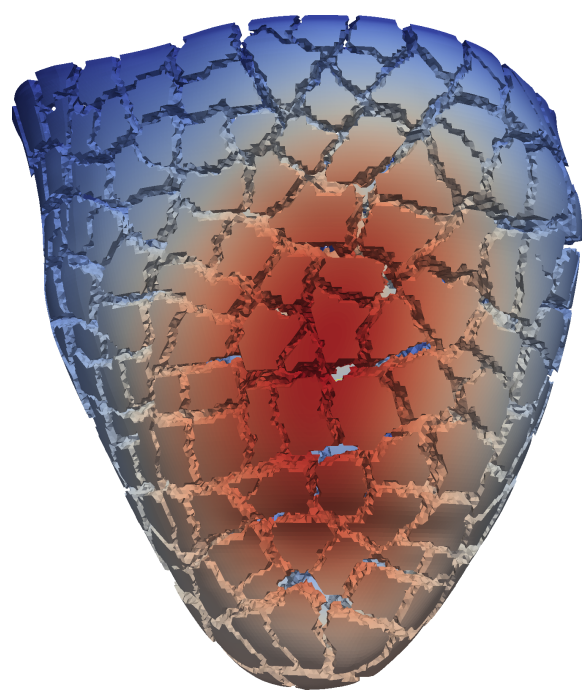

Figure 3: Geometry of a rabbit heart where the colors indicate the displacement field generated by applying a pressure to the inner wall of the left ventricle. Additionally the splits show the decomposition of the mesh into 480 subdomains.

\section{Acknowledgement}

This work was supported by the Austrian Science Fund (FWF) and by the Medical University of Graz within the SFB Mathematical Optimization and Applications in Biomedical Sciences. Additionally, the authors would like to thank A. Prassl, O. Steinbach, G. Holzapfel, G. Haase, M. Liebmann, A. Neic and C. Pechstein.

\section{Bibliography}

[1] G. Holzapfel and R. Ogden, "Constitutive modelling of passive myocardium: a structurally based framework for material characterization," Phil. Trans. R. Soc. A, vol. 367, pp. 3445-3475, 2009.

[2] "Simulating patterns of excitation, repolarization and action potential duration with cardiac bidomain and monodomain models," Mathematical Biosciences, vol. 197, no. 1, pp. 35 - 66, 2005.

[3] T. Eriksson, A. Prassl, G. Plank, and G. Holzapfel, "Influence of myocardial fiber/sheet orientations on left ventricular mechanical contraction. mathematics and mechanics of solids," in press.

[4] C. Augustin and O. Steinbach, "FETI methods for the simulation of biological tissues," in Domain Decomposition Methods in Science and Engineering $X X$ (R. Banks, M. Holst, O. Widlund, Xu, and J., eds.), vol. 91 of LNCSE, Springer, 2013.

[5] A. Neic, M. Liebmann, E. Hoetzl, L. Mitchell, E. Vigmond, G. Haase, and G. Plank, "Accelerating cardiac bidomain simulations using graphics processing units," Biomedical Engineering, IEEE Transactions on, vol. 59, no. 8, pp. 2281-2290, 2012. 